\title{
Analyzing Indoor Environment Sensing Data for Recognizing ADLs of One Person Household
}

\author{
Long Niu ${ }^{1, a, *}$, Sachio Saiki ${ }^{1, b}$ and Masahide Nakamura ${ }^{1, c}$ \\ ${ }^{1}$ Graduate School of System Informatics, Kobe University, 1-1 Rokkodai Nada, Kobe, Japan \\ a longniu@ws.cs.kobe-u.ac.jp, ${ }^{\mathrm{b}}$ sachio@carp.kobe-u.ac.jp, ${ }^{\mathrm{c}}$ masa-n@cs.kobe-u.ac.jp
}

Keywords: Environment sensing, Activities of Daily Living, ADLs recognition, Big data, Machine Learning

\begin{abstract}
Pervasive sensing technologies are promising for increasing one-person households $(\mathrm{OPH})$, where the sensors monitor and assist the resident to maintain healthy life rhythm. Towards the practical use, the recognition of activities of daily living (ADL) is an important step. Many studies of the ADL recognition have been conducted so far, for real-life and human-centric applications such as eldercare and healthcare. However, most existing methods have limitations in deployment cost, privacy exposure, and inconvenience for residents. To cope with the limitations, this paper presents a new indoor ADL recognition system especially for OPH. To minimize the deployment cost as well as the intrusions to the user and the house, we exploit an IoT-based environment-sensing device, called Autonomous Sensor Box (SensorBox). Just placed in the house, SensorBox autonomously measures seven kinds of environment attributes, and uploads them to a cloud server. We apply machine-learning techniques to the collected data, and predicts seven kinds of ADLs. We conduct an experiment within an actual apartment of a single user. The result shows that the proposed system achieves the average accuracy of ADL recognition with more than $90 \%$, by carefully developing the features of environment attributes.
\end{abstract}

\section{Introduction}

The growing number of unmarried people and late marriages in developed countries leads to a social issue of one-person households (OPH). In Japan, the number of OPH increasing rapidly. It is estimated that $37.4 \%$ of all households will become OPH in 2030 [1]. Not only in Japan, it is a worldwide phenomenon. In China, there are more than 60 million of Chinese people currently living alone. The number of OPH will increase to 162 million in 2050 [2]. In seven states in USA, the percentage of OPH exceeds $30.3 \%$ in 2015 [3]. According to [4][5], people in OPH easily lose healthy life rhythm, since no one else can take care of the living in OPH. Since the loss of healthy life rhythm often leads to health deterioration, it is essential to maintain the life rhythm especially in the context of OPH. In general, a life rhythm is characterised by activities of daily living (ADL, for short). Typical ADLs in OPH include cooking, working, eating, taking bath, sleeping, etc. If the 
cycle of ADLs becomes very different from the one in a healthy life rhythm, the resident is losing his/her life rhythm. To maintain the life rhythm, one has to keep a regular record of ADLs. However, keeping manual recording requires strong mind and patience.

A convergence of technologies in machine learning and pervasive computing as well as development of sensors and actuators has caused interest in the development of ADLs recognition to emerge. There are several studies using camera or microphone such as [6][7]. However, such system intrusive too many privacy information, which make residents feel that privacy is violated. There are many studies using various sensors or positioning technologies to measure ADLs such as [8][9]. However, these systems require some sensors be embedded into object (such as key, door or carpet) or be worn on the human body, which have some limitations that high cost of configuration and make users feel inconvenient.

To overcome the limitations of the mentioned related works, we proposed a recognizing ADLs of One-person household system by using indoor environment sensing data. In the proposed system, we use an autonomous SensorBox [16], in this paper we simply call it 'SensorBox', which can be easily deployed without cost-intensive infrastructure and configuration labour to collect indoor environment sensing information. For the types of target recognized ADLs, we defined 7 basic ADLs which are the top list of the need for maintaining life. We applying supervised learning algorithms to analysis environment sensors data. In this paper, we will introduce the construct of proposed system and method of processing data.

To evaluate proposed system's accuracy of recognition ADLs, we actually deployed the system at a resident's apartment and collected massive data during 10 days. As a result, in the case of setting the size of fixed-time window for data and using SVC supervised learning algorithm to analysis, the average accuracy of all ADLs achieved over $85 \%$, and accuracy of some ADLs achieved over $98 \%$. From this result, we confirmed that the proposed system can achieve high accuracy recognition more than $85 \%$ when only using one non-intrusive SensorBox.

\section{Related Work}

The ADL recognition is not a brand-new research topic. Since the need of ADL recognition is great, researchers have been studying and developing a number of methodologies to tackle this problem. The approaches to the ADL recognition can be divided roughly into two categories, depending on the type of contextual information analysed. The first category uses multimedia data taken by video cameras or microphone recordings, to capture the daily living directly. The second category uses time-series data measured by various sensors, including accelerometer, gyroscope, RFID, and power-meters sensors.

Multimedia data: Brdiczka et al. [10] video tape smart home residents and process the video to recognize activities. While individuals have traditionally been resisted to at-home video monitoring [11], the acceptance of this technology in the home is increasing. On the other hand, processing the video is very computationally expensive and relies upon first tracking the resident before the correct video data can be captured and analysed [12].

Sensor data: Such first kind of data collection, inside home, is considered intrusive too many and computationally expensive, hence ADLs recognition system using passive information becomes highly applicable. Recently, most current work in ADLs recognition use sensor data. Researchers have found that different types of sensor information are effective for classifying different types of activities.

Kusano et al. [9] proposed a system that derives life rhythm from tracking elderly movement by using RFID positioning technology. They spread RFID equipment on the floor and asked participants to wear slippers with RFID tags reader that recorded indoor location of resident. 
Reasoning life rhythm based on the time-series location data. However, it cannot determine the exact activity only by movement locus, so the accuracy of ADLs recognition is low.

Munguia-Tapia et al. [14] and Philipose et al. [15] observe resident's interaction with object of interest such as doors, windows, refrigerators, keys, and medicine containers. Munguia-Tapia et al. installed state-change sensors on key items to collect object interaction data, while Philipose et al. put RFID tags on items and asked participants to wear gloves with RFID tag readers that recorded when the individual was close to a key item.

Pei et al. [8] combined the positioning technologies and phone sensors to capture human movements in natural environments and use the movements to study behaviour. However, in the case of turning on motion-sensors Wi-Fi and GPS insight of smartphone simultaneously the battery drain rate is very high. On the other hand, users in home may not carry smartphone all time with them, which makes it a partially available source of information.

\section{Challenges and Research Goal}

The ADL recognition has been widely studied for a few years. By keeping track of ADLs, a smart pervasive system can provide reminders for residents, as well as react to hazardous situations [13]. Most of these studies apply to elderly people, cancer patients, and ordinary families. However, there are not so many studies for One-Person-Household (OPH). The unique characteristics of OPH are: the resident is living alone, and is often busy to do everything by oneself. He/she does not want to change the own way of living, or pay for expensive systems just for monitoring ADLs.

As mentioned in Section 2, there are many existing systems that use wearable sensors, objectembedded sensors, or indoor positioning systems. However, we consider it difficult for people in $\mathrm{OPH}$ to accept these technologies, because they are too exaggerated and intrusive for their life. We can easily imagine that most residents will forget or give up wearing the sensor, since the home is the place where the resident make oneself comfortable. Although labs or companies can manage the large-scale equipment, it is still too expensive to deploy in $\mathrm{OPH}$.

Our research goal is to minimize such limitations of the conventional approaches, and to achieve high-quality ADL recognition of $\mathrm{OPH}$.

\section{System Architecture}

In order to achieve our research goal, we proposed an ADLs recognition System for one-person household by learning indoor environment sensing data. The architecture of proposed system be shown in Figure 1. As shown in figure, the development of system and processing of data take a step-by-step approach from left to right.

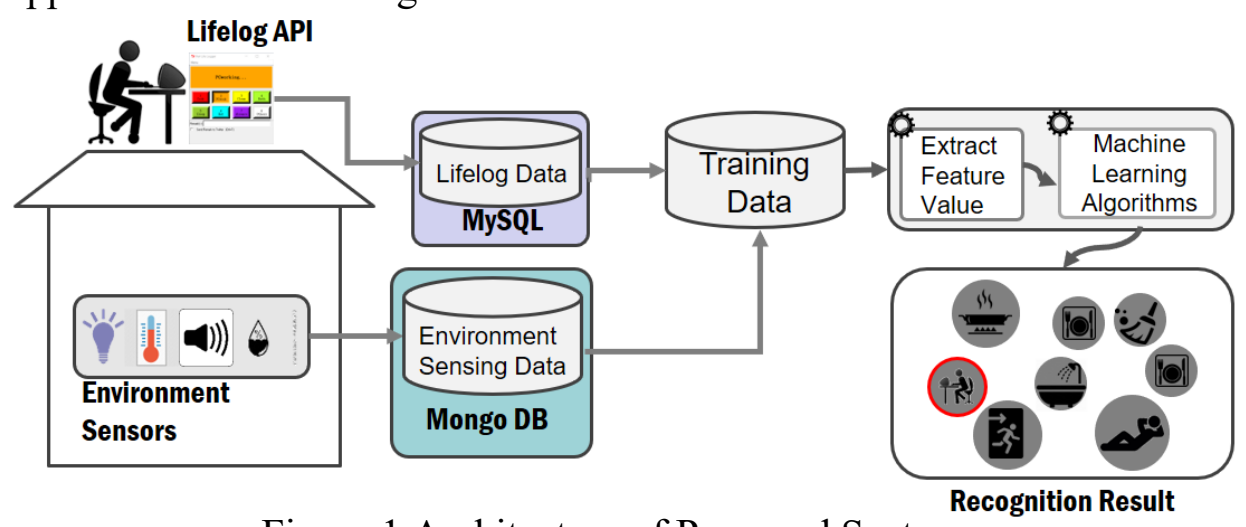

Figure 1 Architecture of Proposed System 
First, setting up experiment environment at testbed, which include installing an equipment to collect indoor environment sensing data (such as, temperature, sound volume, light etc.) and installing an API on residents' PC or smartphone to collect the really ADLs record which be used to label training data with real ADLs of OPH. Then system begin to collect data: resident's real ADLs $\log$, which will be stored in MySQL database, and indoor environment sensing data, which will be stored in Mongo database.

The next step is data analysis. The process of data analysis consists of three detailed steps: establish training data, extraction of feature value and construction of machine learning algorithm.

Hereinafter, each subsection will describe the three steps in detail.

\subsection{Experiment Setup and Data Collection}

The testbed that we are using to validate our system is an actually resident's apartment. As shown in Figure 2, the apartment testbed is an ordinary single apartment in Japan, which includes one bed/living room, one bathroom and kitchen. The apartment is equipped with the SensorBox in kitchen room. In the figure 2, the red square showing the position of SensorBox deployed in room.

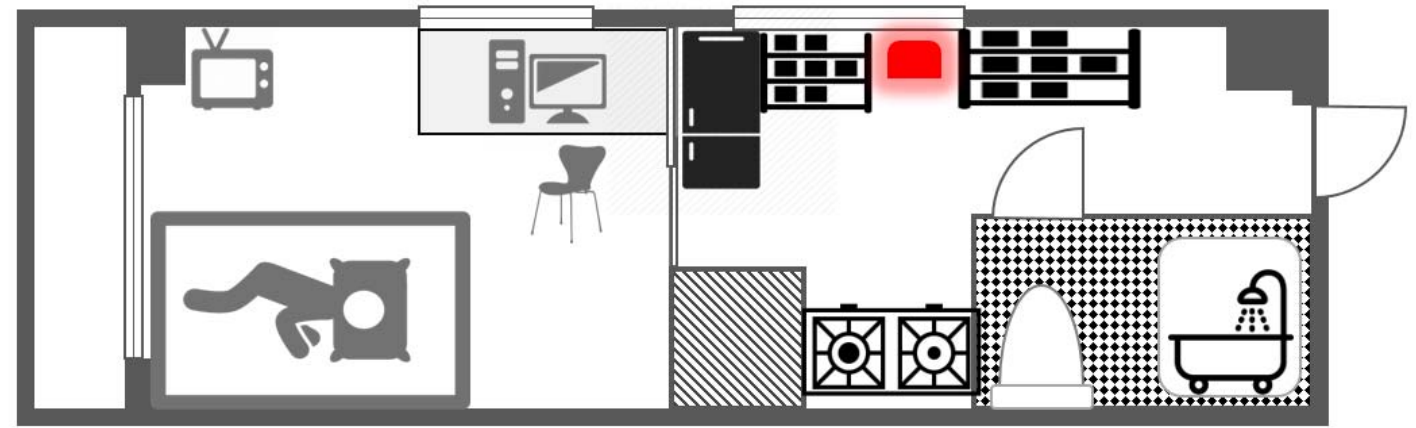

Figure 2 Apartment of Testbed, position of SensorBox

The sensor equipment was developed by our research group [16], which measures various indoor environmental data by using environment sensors included temperature, humidity, lighting intensity, atmosphere pressure, sound volume, human motion and vibration. In additional, we developed an indoor environment sensing service using autonomous SensorBox that can be easily deployed without cost-intensive infrastructure and configuration labour. Figure 3 shows its physical form. Logger in SensorBox, in every time of measurement, create data and modify to JSON formal text, then upload to the Mongo Database.

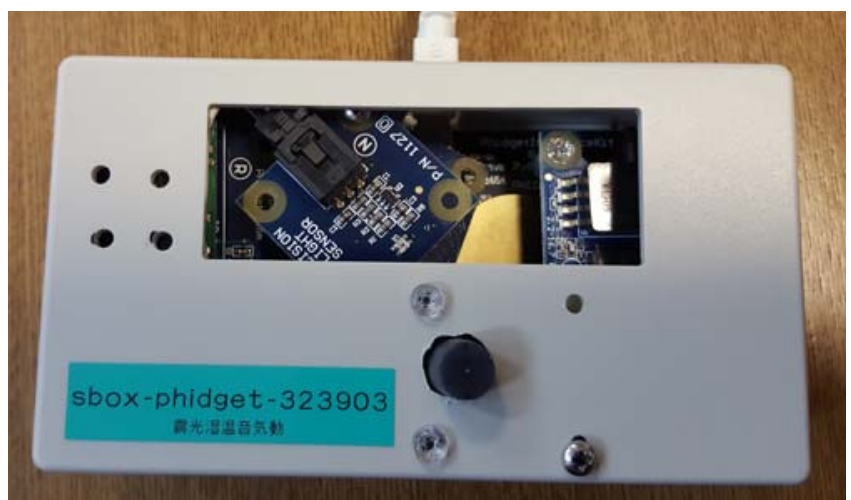

Figure 3 SensorBox

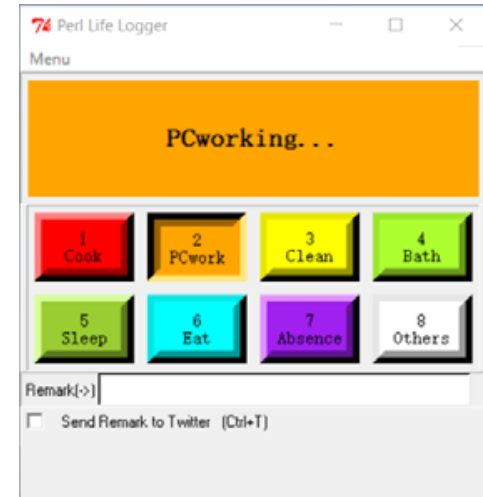

Figure 4 Lifelog tools UI

For supervised learning, the system needs to train data that have the correspondence between the ADLs and sensors data in advance. To label the sensor data, we developed a API, which we will 
call it 'Lifelogger'. Figure 4 shows the users interface of API. The operation of the API is very simple. User only needs to click the button corresponding to the real behaviour when they changing their activity. Reference related studies [5][6], we defined 8 types of main ADLs within one day at home: sleep, eat, cook, working at PC, clean, bath, absence and other.

We collected massive environment sensing data from a OPH's home during 10 days. We have been collected 45693 sets of raw sensors data with filtering the training data labelled 'other' that is outside of the range of system's recognizing ADLs.

\subsection{Data Manipulation and Analysis}

In our research, we employ supervised learning method to recognize indoor ADLs of OPH. The process of recognition method is composed of 3 main steps as follows.

Establish Training Data of ADLs: For supervised learning, the system needs to training data which have the correspondence between the ADLs and data in advance. In order to establish training data, we integrate the sensors log and ADLs log by referencing the time attribute of two datasets. The training data store each sensor log by attached activity label.

Extraction of Feature Value: Feature value is data that is effective to identify the ADLs. In this step, we get the feature value from training data, as the following process. First, extract sensor data that are susceptible to changes in ADLs of resident, such as sound volume, lighting intensity etc. Then, integrating raw sensor data within fixed-time size window. The size of the window affects accuracy - too large of a window will likely include sensor events from a different activity, while too small of a window will not provide sufficient data to the machine learning algorithm. We experimented with models that used window sizes of 1, 2 and 3 minutes. The integration algorithm of each type of sensor data is different. Due to protect patent, we do not elaborate on each sensor integration algorithm.

Construction of Recognition Model: Based on the feature value of every ADL, construct a machine learning model. There are some popular pattern recognition algorithms, such as SVC, Decision Tree and Random Forest. By using the algorithm, given data can be classified into one of ADLs.

\section{Experimental Results}

In this section, we will show the results of the analysis based on data collected in 10 days. We set different size of fixed-time widow, 1/2/3 minutes, to observe the impact of the size of time widow on the accuracy prediction. And we applied three different machine learning algorithms for each data to predict ADLs. The results of our assessment experiment, summarized in Table 1, were consistent with our expectations. For machine learning algorithms, we used 'sklearn' library of python program.

From the results shown in Table 1, we find that when the size of time widow is set on 2 minutes, the accuracy of each algorithm reached the highest accuracy of ADLs recognition. The result verifies the content mentioned at subsection 4.2 that the size of time window should not be too large or too small.

For the detail accuracy of each ADLs recognition, we discussion it based on a representative result that when size of time window is 1 minute and algorithm is SVC. Table 2 shows the detail accuracies of each ADLs recognition. From table 2, we can clearly find two ADLs, sleep and absence, are relatively high. In addition, the proportion of the two ADLs of data sets are also particularly high. In order to verify whether the average accuracy of all ADLs is affected by the two ADLs sleep and absence. We reanalysed the data, after filtered out the datasets labelled with the 2 
types of ADLs. The results are shown in Table 3. The results of reanalysed show that the accuracy of other ADLs recognition and average of all ADLs recognition slightly improved, which beyond our expectation.

Through the results of experiment, we got the conclusion that by analysing indoor environment sensing data can predict the ADLs of One-person household with relatively accuracy of ADLs recognition.

Table 1 Average accuracies of all ADLs of each dataset and algorithm

\begin{tabular}{|c|c|c|c|}
\hline & $1 \mathrm{~min}$ & $2 \mathrm{~min}$ & $3 \mathrm{~min}$ \\
\hline SVC & $87.32 \%$ & $88.73 \%$ & $88.17 \%$ \\
\hline Decision Tree & $85.83 \%$ & $86.11 \%$ & $85.42 \%$ \\
\hline Random Forest & $87.32 \%$ & $88.73 \%$ & $88.17 \%$ \\
\hline No. of datasets & 7200 & 3800 & 2400 \\
\hline
\end{tabular}

Table 2 Accuracies of all ADLs of 1 minutes \& SVC

\begin{tabular}{|c|c|c|c|c|}
\hline & precision & recall & F1-core & Support \\
\hline Cook & $66 \%$ & $99 \%$ & $79 \%$ & 185 \\
\hline Working at PC & $99 \%$ & $26 \%$ & $41 \%$ & 546 \\
\hline Clean & $39 \%$ & $98 \%$ & $56 \%$ & 200 \\
\hline Bath & $69 \%$ & $88 \%$ & $77 \%$ & 42 \\
\hline Sleep & $98 \%$ & $100 \%$ & $99 \%$ & 1735 \\
\hline Eat & $97 \%$ & $78 \%$ & $87 \%$ & 240 \\
\hline Absence & $98 \%$ & $100 \%$ & $99 \%$ & 762 \\
\hline Avg / total & $93 \%$ & $87 \%$ & $86 \%$ & 3700 \\
\hline
\end{tabular}

Table 3 Accuracies of all ADLs of 1 minutes \& SVC, filtered sleep \& absence

\begin{tabular}{|c|c|c|c|c|}
\hline & precision & recall & F1-core & Support \\
\hline Cook & $95 \%$ & $100 \%$ & $97 \%$ & 96 \\
\hline Working at PC & $99 \%$ & $98 \%$ & $99 \%$ & 144 \\
\hline Clean & $89 \%$ & $95 \%$ & $92 \%$ & 121 \\
\hline Bath & $44 \%$ & $83 \%$ & $58 \%$ & 24 \\
\hline Eat & $100 \%$ & $81 \%$ & $90 \%$ & 170 \\
\hline Avg / total & $94 \%$ & $92 \%$ & $92 \%$ & 555 \\
\hline
\end{tabular}

\section{Conclusion}

In this paper, we have proposed a system recognizing ADLs of OPH by only using environment sensors, to achieve high accuracy of recognition and greatly reducing the cost of implement for residents. To evaluate the accuracy of ADLs recognition, we deployed the proposed system at one resident's apartment and collect environment sensing data in 10 days. As a result of extracting feature value of data, the average accuracy of all ADLs achieved more than $85 \%$, for some ADLs, sleep and absence, the accuracy of recognition achieved more than $90 \%$.

As for the future work, we plan to deploy the system on the web service and apply it to multiresident. In addition, the proposed system can recognize limited ADLs, we need to extend the types of ADLs recognizable. 


\section{Acknowledgements}

This research was partially supported by the Japan Ministry of Education, Science, Sports, and Culture [Grant-in-Aid for Scientific Research (B) (No.16H02908, No.15H02701), Challenging Exploratory Research (15K12020)], and Tateishi Science and Technology Foundation (C) (No.2177004).

\section{References}

[1] L. Ministry of Health and Welfare, “Annual health, labour and welfare report 2008-2009,” 2010.

[2] Percentage of single-person households in the U.S. in 2015, by state. https://www.statista.com/statistics/, 2017

[3] Living Alone in China: Projection of One-person Household, 2010 to 2050, from https://paa.confex.com/

[4] Asoka. S., Fukuda, K. and Yamazaki, K. (2004) Effects of sleep-wake pattern and residential status on psychological distress in university students. Sleep and Biological Rhythms, 2 (3) :192-198

[5] Fujino, Y., Iso, H., Inaba, Y., Koizumi, A., Kubo, T. for the Japanese Collaborative Cohort Study Group (2006) A Prospective Cohort Study of Shift Work and Risk of Ischemic Heart Disease in Japanese Male Workers. Am. J. Epidemiol., 164: 128 - 135

[6] Fiore, L., Fehr, D., Bodor, R., Drenner, A., Somasundaram, G., and Papanikolopoulos, N. "Multi-camera human activity monitoring," Journal of Intelligent and Robotic Systems, vol.52, no.1, pp.5-43, 2008.

[7] K.O.M. Doi, "Smartphone-based monitoring system for activities of daily living for elderly people and their relatives etc.," Proceedings of the 2013 ACM Conference on Pervasive and Ubiquitous Computing Adjunct Publication, UbiComp '13 Adjunct, pp.103-106, ACM, 2013.

[8] L. Pei, R. Guinness, R. Chen, J. Liu, H. Kuusniemi, Y. Chen, L. Chen, and J. Kaistinen, "Human behavior cognition using smartphone sensors," Sensors, vol.13, no.2, pp.1402-1424, 2013.

[9] Kusano, K. Muro, H. Hayashi, T. Harada, F. and Shimakawa, H. "Derivation of life rhythm from tracing elderly movement," The $10^{\text {th }}$ Forum on Information Technology, FIT2011, pp.891-892, 2011

[10] O. Brdiczka, P. Reignier, and J. Crowley. Detecting individual activities from video in a smart home. Proceedings of the International Conference on Knowledge-Based and Intelligent Information and Engineering Systems, 2007, pp. 363-370.

[11] B.K. Hensel, G. Demiris, and K.L. Courtney. Defining obtrusiveness in home telehealth technologies: A conceptual framework. Journal of the American Medical Informatics Association, 13(2006), 428-431

[12] C. Stauffer and E. Grimson. Learning patterns of activity using real-time tracking. IEEE Transactions on Pattern Analysis and Machine Intelligence, 22(8):747-757, 2000

[13] C. Wren and E. Munguia-Tapia. Toward scalable activity recognition for sensor networks, Proceedings of the Workshop on Location and Context-Awareness, 2006, 218-235

[14] E. Munguia-Tapia, S.S. Intille, and K. Larson. Activity recognition in the home using simple and ubiquitous sensors. Proceedings of PERVASIVE, 2004, pp. 158-175.

[15] M. Philipose, K. Fishkin, M. Perkowitz, D. Patterson, D. Fox, H. Kautz, and D. Hahnel. Inferring activities from interactions with objects. IEEE Pervasive Computing, 3(2004), 50-57.

[16] S. Sakakibara, S. Saiki, M. Nakamura, and S. Matsumoto, "Indoor environment sensing service in smart city using autonomous SensorBox," 15th IEEE/ACIS International Conference on Computer and Information Science (ICIS 2016), pp.885-890, 2016. 